\title{
Improved Diffusion Basis Functions Fitting and Metric Distance for Brain Axon Fiber Estimation
}

\author{
Ramón Aranda ${ }^{1}$, Mariano Rivera ${ }^{1}$, and Alonso Ramírez-Manzanares ${ }^{2}$ \\ 1 Centro De Investigación en Matemáticas, Guanajuato, Gto, México, 36240 \\ 2 Universidad de Guanajuato, Departamento de Matemáticas, Guanajuato, Gto, \\ México, 36240 \\ arac@cimat.mx, mrivera@cimat.mx, alram@cimat.mx
}

\begin{abstract}
We present a new regularization approach for Diffusion Basis Functions fitting to estimate in vivo brain the axonal orientation from Diffusion Weighted Magnetic Resonance Images. That method assumes that the observed Magnetic Resonance signal at each voxel is a linear combination of a given diffusion basis functions; the aim of the approach is the estimation of the coefficients of the linear combination. An issue with the Diffusion Basis Functions method is the overestimation on the number of tensors (associated with different axon fibers) within a voxel due to noise, namely, the over fitting of the noisy signal. Our proposal overcomes such an overestimation problem. In additionally, we propose a metric to compare the performance of multi-fiber estimation algorithms. The metric is based on the Earth Mover's Distance and allows us to compare in a single metric the orientation, size compartment and the number of axon bundles between two different estimations. The improvements of our two proposals is shown on synthetic and real experiments.
\end{abstract}

\section{Introduction}

Water diffusion estimation has extensively been used in recent years as an indirect way to infer axon fiber pathways and this, in turn, has made the estimation of fiber connectivity patterns in vivo one of the most challenging goals in neuroimaging. For this purpose, a special Magnetic Resonance Imaging (MRI) technique named Diffusion Weighted MRI (DW-MRI) is used. This imaging technique allows one to estimate the preferred orientation of the water diffusion in brains which, in the white matter case, is usually constrained along the axon orientations. This information is very useful in neuroscience research due to changes that occur in the neural connectivity patterns with neurological disorders and, in general, with brain development [12].

The water diffusion angular variation, within cerebral tissue, has been summarized in most medical applications by Diffusion Tensor Magnetic Resonance Images (DT-MRI) 34. In the case of brain images, DT-MRI produces a tensor field that indicates the local orientation of nerve bundles. The local orientation of the corresponding nerve bundle is estimated from the orientation of 


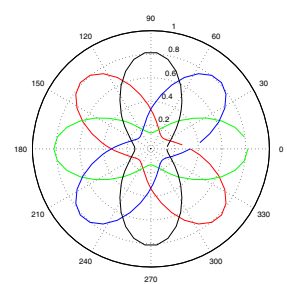

(a)

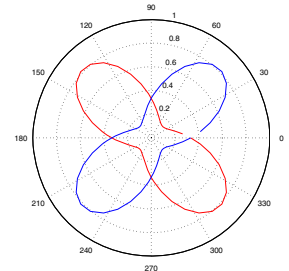

(b)

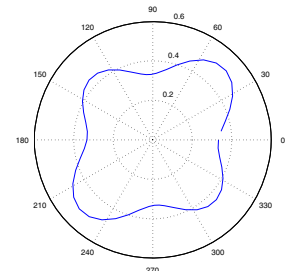

(c)

Fig. 1. 2D scheme of the diffusion basis functions, the narrow part of the signals correspond to the orientation of the diffusion peaks. (a) Set of 4 DBFs, (b) Subset of two DBFs, (c) Signal obtained by adding the two DBFs of (b).

the first eigenvector of a tensor, the one associated with its largest eigenvalue. This orientation is known as the Principal Diffusion Direction (PDD) [5]. In [6] Stejskal-Tanner presented a mono-exponential model of the decayed MR signal. Nowadays, it is well known that this technique is limited by the partial volume effect; i.e. diffusion at voxels with crossing fibers or bifurcations can not be represented by a single tensor.

Given the evident difficulty of DT-MRI for dealing with more than one diffusion direction per voxel, there has been proposed more sophisticated parametric models; see for instance refs. [789]. In particular, Multi-Tensor Diffusion MRI (MTDMRI) can deal with fibers that split, merge or cross [7]. For instance, Tuch et.al [10] proposed to use the Gaussian Mixture Model (GMM). This model explains better the diffusion phenomenon for two or more fibers in a given voxel $k$, as:

$$
S_{i, k}=S_{0, k} \sum_{j=1}^{J} \beta_{j, k} \exp \left(-b g_{i}^{T} T_{j, k} g_{i}\right),
$$

where $S_{0, k}$ is the signal without diffusion, $b$ is a constant acquisition parameter, $g_{i}=\left[g_{x_{i}}, g_{y_{i}}, g_{z_{i}}\right]^{T}$ is a unitary vector which indicates the direction on which is applied an independent magnetic gradient, with $i=1,2, \ldots M$ and $M$ is the total number of applied gradients; $T_{j, k}$ is the $j$-th tensor, a $3 \times 3$ symmetric positive definite matrix; $S_{i, k}$ is the DW-MR signal measured when $g_{i}$ was applied. $\beta_{j}$ is the contribution of tensor $T_{j, k}$ and it is constrained by $\beta_{j} \in[0,1]$ and $\sum_{j=1}^{J} \beta_{j}=1$. Finally, $J$ indicates the total number of tensors within the voxel. Thus, equation (11) shows the relationship between the signal without diffusion and the signal with diffusion on the direction $g_{i}$. Solving (11) is computationally expensive because it leads to a non linear optimization problem. Note that it requires to first estimate the number of axon bundles, $\mathrm{J}$ (solve the model selection problem), then to estimate all the others unknowns.

In [1] the authors proposed a strategy for solving the inverse problem stated in (1). They avoided the non-linear optimization problem by using a fixed set of Diffusion Basis Functions (DBF). The basis dictionary is, by definition, incomplete because it is computed on a discretization of the orientational 3D space. Then, the DW-MRI is approximated at each voxel by a linear combination of DBFs as it is shown in Figure 1 . 


$$
S_{i, k}=\sum_{j=1}^{N} \alpha_{j} \phi_{i, j},
$$

with $\alpha_{j} \geq 0$, where the $j-t h \mathrm{DBF}$ is defined by

$$
\phi_{i, j}=S_{0, k} e^{\left(-b g_{i}^{T} \bar{T}_{j} g_{i}\right)} .
$$

The coefficient $\phi_{i, j}$ can be understood as the diffusion weighted signal corresponding to the gradient $g_{i}$ when a single perfect fiber is oriented with the largest engenvector of the base (fixed) tensor $\bar{T}_{j}$. Based on this approach, the GMM model (1) is transformed to a programming (optimization) problem. In particular, the non-negative least-squares (NNLS) formulation corresponds to 12]13:

$$
\begin{array}{ll}
\min _{\alpha \in \mathbb{R}^{N}} & \|\Phi \alpha-S\|_{2}^{2} \\
\text { subject to } & \alpha \geq 0
\end{array}
$$

where $\Phi=\left\{\phi_{i, j}\right\}_{i=1,2, \ldots M, j=1,2, \ldots N}$ and $\alpha=\left[\alpha_{1}, \alpha_{2}, \ldots, \alpha_{N}\right]^{T}$ is the unknown vector of the linear system. It has been reported that this NNLS based DBF approach is prone to overestimate the number of fibers [14].

\section{Sparse Diffusion Basis Functions}

In this section, we propose a new regularization method for the DBF fitting to recover axonal intra-voxel information. As mentioned before, the solver in (4) is prone to recover more basis tensors than the actual number of tensors, that is to say, vector $\alpha$ is not so sparse. However, we note in our experiments that the solution given by solving (4) is close to the actual one. In the following, we say that $\alpha_{j}$ is active if $\alpha_{j}>0$ and inactive if $\alpha_{j}=0$, in other words, $\alpha_{j}$ is active if it contributes to the linear combination in (2). By experience, we know that the number of axon bundles within a voxel is small (1,2 or 3) so that $\alpha$ must be sparse; i.e. the signal is approximated with a reduced number of component base signals. Thus, we propose to solve:

$$
\begin{array}{ll}
\min _{c \in \mathbb{R}, \alpha \in \mathbb{R}^{N}} & \|c \Phi \alpha-S\|_{2}^{2}+\lambda\|\alpha\|_{2}^{2} \\
\text { subject to } & \alpha \geq 0,
\end{array}
$$

using as starting point the solution given by (4); i.e., the solution computed with $\lambda=0$.

In our approach, sparse $\alpha$ vectors are promoted by the combined effect of: the second regularization term in (5), the non-negativity constraint and the scale factor $c$. The $c$ parameter scales the solution, so that the error is reduced. The solution is computed by alternating the minimization with respect to (w.r.t.) $\alpha$ and $c$. In particular, the minimization w.r.t. $\alpha$ is achieved with a Gauss-Seidel scheme, where the non-negativity constraint is satisfied by projecting to zero 
the negative $\alpha_{j}$ values in each iteration. This has the drawback that it requires several iterations to compute the optimum $\alpha$. To accelerate the convergence, we iterate the Gauss-Seidel solver a few times alternated with a subspace minimization strategy [15]. On the other hand, the minimization w.r.t. $c$ leads us to the close formula:

$$
c=\frac{(\Phi \alpha)^{T} S}{\alpha^{T} \Phi^{T} \Phi \alpha} .
$$

Then, in order to keep the $\alpha$ values in a reasonable scale and $c$ close to one, we renormalize the $\alpha$ vector at each iteration:

$$
\alpha \leftarrow \frac{1}{c} \alpha
$$

\section{Multi-Axon Fiber Recovering Metric}

One of the problems in the DW-MRI analysis is the definition of a metric that reflects all the aspects of the multi-axon bundle estimation: to estimate the number of fibers, their orientations and their compartment sizes. Several metrics can be used for measure each one of the mentioned aspects, see for instance [14. However, in the best of our knowledge, there is not a metric that resumes in a scalar value (and thus makes directly comparable different multi-fiber methods) the performance of the fiber estimation methods. Here we propose a new metric for this aim.

First the observed DW-MR signal is an effect of the water diffusion. Such actual diffusion directions are close to some directions represented in the basis functions (see Figure 2). Then, talking about synthetic data, given the observed noisy signal (Panel 2(c)), one wants to recover the fiber information (Panel $2(\mathrm{a})$; i.e., we want to minimize the error between the recovered fibers and the actual fibers. Hence, we need to compare the different axon fiber features shown in panels 2(a) and 2(e). Thus, it is necessary to define an error measure that involves those features. However, it is common to use as a quality measure the error between the restored signal, $S_{\text {rest }}$ (Panel 2(d)], and the actual noiseless signal, $S_{a c t}($ Panel 2(b)):

$$
\text { error }=\left\|S_{a c t}-S_{\text {rest }}\right\|^{2}
$$

We claim that the correct error metric is the error between the estimated fiber's information in Panel 2(e) and the actual one in Panel 2(a) (one important fiber feature is its orientation). Unfortunately, we have the fiber's information separated. According with 14, we first need to match the fibers which are closer, and then measure three different errors:

- The angular error between the actual and the estimated fiber.

- The magnitude of the difference of the relative diffusion size (size compartment).

- The difference between the number of fibers. 


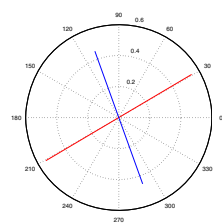

(a)

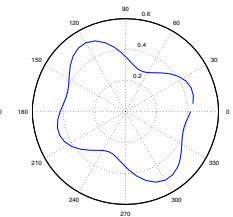

(b)

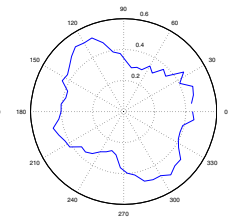

(c)

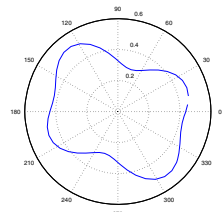

(d)

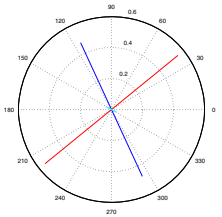

(e)

Fig. 2. 2D illustration of the diffusion in a voxel with a crossing axon fiber. (a) Actual fiber orientations, (b) original noiseless signal, (c) noisy observed signal, (d) restored signal and (e) estimated axon fibers.

Nevertheless, it is not clear how to weight and integrate all that information in a single metric.

In the following, we propose a new error measure that considers the fiber's size compartments as discrete distributions. Our measure takes into account important features of the fibers: the density distribution (shape), the total diffusion and the fiber orientation.

\subsection{A Metric for Multi-Fiber Estimations}

Here, we propose a metric to measure the error between the recovered multifiber solution and the Ground Truth (GT). Before to formally define our metric, it is important to introduce the relevant features of the axon fibers within a voxel.

Let $\beta=\left\{\beta_{1}, \beta_{2}, \ldots, \beta_{J}\right\}$ and $T=\left\{T_{1}, T_{2}, \ldots, T_{J}\right\}$ be the actual values (the GT) used for generating synthetic signal by using the equation (1), so that $v_{i}$ is the largest eigenvector of $T_{i}$ (the PDD) that is associated with the fiber orientation; then we propose to compute the error between the GT fiber distribution, represented by the vectors $\beta$ and $v$, and the estimated one represented by their compartments $\alpha$ and their orientation vectors $\tilde{v}$ with a metric of the form:

$$
D_{F}([\beta, v] ;[\alpha, \tilde{v}])=D_{o}([\beta, v] ;[\alpha, \tilde{v}])+D_{v}(\beta ; \alpha)+D_{s}(\beta ; \alpha) .
$$

Each term in (9) is explained in the following. Note that the number of elements in $\beta$ and $\alpha$ is different. The same case occurs with $v$ and $\tilde{v}$.

Orientation Term: $\boldsymbol{D}_{\boldsymbol{o}}$. Given two fibers, with PDDs $v_{i}$ and $\tilde{v}_{j}$, we define the angle $[0, \pi]$ between the PDDs as orientation distance:

$$
d\left(v_{i}, \tilde{v}_{j}\right)=\arccos \left(v_{i} \cdot \tilde{v}_{j}\right) .
$$

From now on, we denote $d_{i j}=d\left(v_{i}, \tilde{v}_{j}\right)$. Then, we can see $d_{i j}$ as the cost (measure of error) of saying that $\tilde{v}_{j}$ is the orientation of a single fiber when the real orientation is $v_{i}$. In our problem we can have several fibers, so we need to match the corresponding compartment in order to find the cost. Such a match must be the one that computes the minimum cost; i.e. we need to match the closest 
fibers. Hence, the computation of this metric is formulated as the solution of the transportation problem:

$$
\begin{aligned}
& D_{o}([\beta, v] ;[\alpha, \tilde{v}])=\min _{x} \frac{\sum_{i, j} d_{i j} x_{i j}}{\sum_{i, j} x_{i j}} \\
& \text { subject to } \\
& \sum_{j} x_{i j} \leq \beta_{i}, \\
& \sum_{i} x_{i j} \leq \alpha_{j} \\
& x_{i j} \geq 0 \\
& \sum_{i, j} x_{i j}=\min \left\{\sum_{i} \beta_{i}, \sum_{j} \alpha_{j}\right\} .
\end{aligned}
$$

In this manner, $x_{i j}$ denotes the transportation flows and represents the amount transported from the $i-t h$ supply to the $j-t h$ demand.

The system of equations (11) is known as the Earth Mover's Distance (EMD). The EMD is a measure which evaluates the dissimilarity between two multidimensional distributions in some feature space by using a distance measure between single features. This distance is defined as the minimal cost that must be paid to transform one distributions into the other [16]17.

Total Diffusion Term: $\boldsymbol{D}_{\boldsymbol{v}}$. The term $D_{v}$ measures the distance between the total diffusion of the estimated fiber and the GT, we use:

$$
D_{v}(\beta ; \alpha)=c_{v}\left|\sum_{i} \beta_{i}-\sum_{j} \alpha_{j}\right| .
$$

where $c_{v}$ is a positive scalar that weights the contribution of the term. In order to make comparable the contribution of this term w.r.t the previous term, $D_{o}$, a choice for $c_{v}$ is:

$$
c_{v}=\max _{i, j}\left\{d_{i j}\right\}
$$

where $d$ is defined in (10). The combination of $D_{o}$ and $D_{v}$ using this particular selection for $c_{v}$ was previously reported by Pele and Werman for comparing unnormalized image color histograms [18].

Sparsity Term: $\boldsymbol{D}_{\boldsymbol{s}}$. The last term $D_{s}$ measures the difference between the sparsity of the the GT and the estimation. Let

$$
N(\beta)=\frac{\beta}{\sum_{k} \beta_{k}}
$$

be the normalization operator. Thus, we see $N(\beta)$ and $N(\alpha)$ as discrete density probability functions (for an orientation distribution) and compare their sparsity by comparing their Shannon's entropy: 


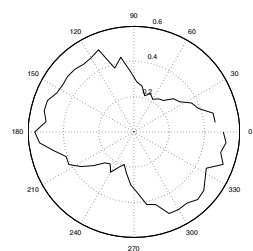

(a)

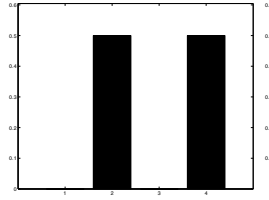

(d)

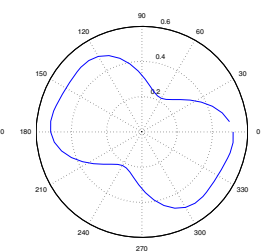

(b)

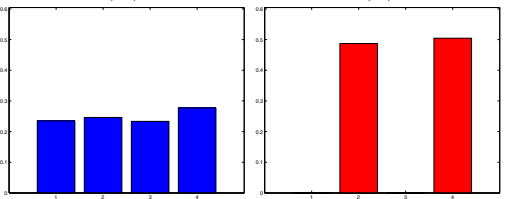

(e)

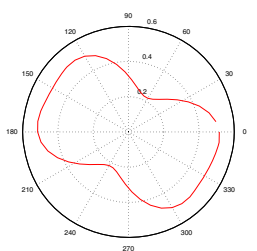

(c)

(f)

Fig. 3. 2D example of the estimation of $\beta$ coefficients by using the equations (4) and (5). The noisy signal (a) was built with the $\beta$ coefficients showed in (d), noise with Rician distribution was added. (b) and (e) show the restored signal and the computed basis coefficients with (4). (c) and (f) show the restored signal and the computed basis coefficients by our proposal in (5). Note that, even the recovered signals are similar, the new methodology does not overestimate the number of axon bundles.

$$
D_{s}(\beta ; \alpha)=c_{s}\left|\sum_{i} N(\beta)_{i} \log N(\beta)_{i}-\sum_{j} N(\alpha)_{j} \log N(\alpha)_{j}\right|
$$

where $c_{s}$ is a parameter that keeps the value of $D_{s}$ comparable with $D_{o}$ and $D_{v}$; we use:

$$
c_{s}=\max \left\{\sum_{i} \beta_{i}, \sum_{j} \alpha_{j}\right\} \text {. }
$$

\section{Experiments and Results}

In this section we present the results obtained on synthetic DW-MRI and real DW-MRI data.

\subsection{Synthetic 2D Example}

First, we show a 2D example to illustrate the methodology. Figure 3 shows the results obtained by solving the equations (4) and our proposal in (5). Note that in this case the observed signal was built as a linear combination of the signals basis functions (this is not always the case, but allows to sintheticaly illustrate our proposal) and noise with Rician distribution was added. As mentioned before, the values of the size compartment are visualized as a discrete distribution. One can see that solution by using (4) gives more signal basis than the solution by solving (5) and both restored signals are similar. The overestimation problem on formulation (4) is because the signal fitting does not penalize the number of 


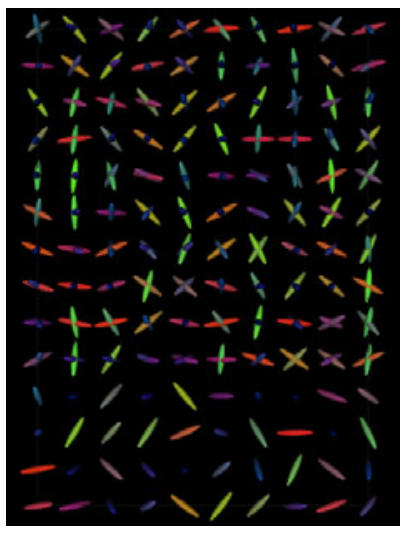

(a)

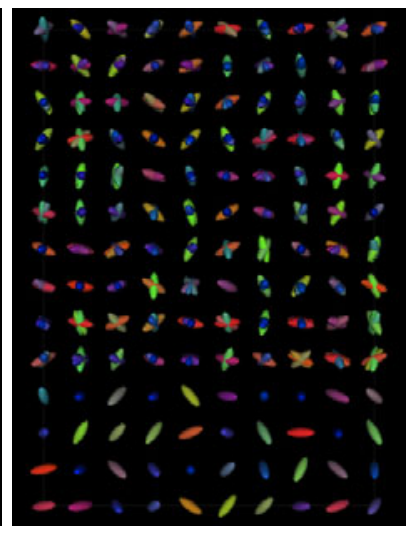

(b)

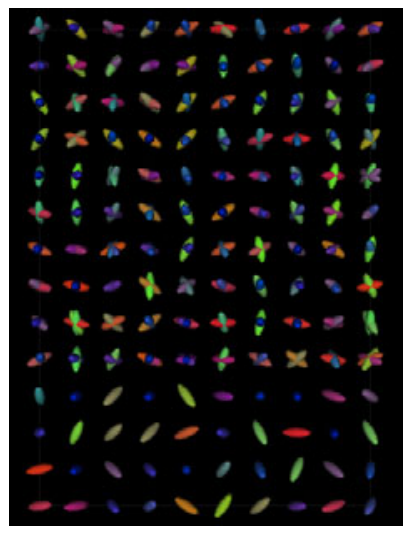

(c)

Fig. 4. Results on synthetic data with $S N R=10$. (a) actual tensors, (b) estimation using the equation (4) and (c) estimation using our proposal in equation (5).

coefficients and only minimizes the difference between signals, as opposite that in our formulation.

Moreover, the proposed error measure $D_{F}$ detects the difference between the two solutions: the error with respect to the GT for the solution in Panel 3(e) and $3(\mathrm{f})$ are 0.5592 and 0.0137 , respectively. Thus, our metric indicates that the proposed sparse solution is closer to the GT. Note how our metric allows to detect differences and similarities between all the features of axon multi-fiber solutions by the evaluation of a single metric.

\subsection{Synthetic 3D Example}

In this experiment, we used 3D synthetic data with the following description:

- Synthetic data. The DW-MRI signal was synthesized from the GMM (1). The DT principal eigenvalue was set to $1 \times 10^{-3} \mathrm{~mm}^{2} / \mathrm{s}$ and the second and third tensor eigenvalues were $2.22 \times 10^{-4} \mathrm{~mm}^{2} / \mathrm{s}, \mathrm{FA}=0.74$. The above values were taken from a sample of tensors observed in the brain data from a healthy volunteer. The tensors were randomly rotated to generate a composed field of 140 voxels. Rician noise was added to each measurement to produce a low Signal to Noise Ratio (SNR) equal to 10, which is typically found on clinical settings.

Now, in Figure 4 we show the results obtained by using the synthetic data. The Panel 4(a) shows the GT. Note that the synthetic data represent two cases: voxels with crossing fibers and voxels with single fiber. We can see that formulation (5) gives a closer solutions to the GT w.r.t. the formulation in (4). Moreover, Figure 5 shows the number of tensors in each voxel for the GT and the two formulations. One can see that the number of tensors computed by formulation in (5) is closer to the GT, i.e. it is more sparse and eliminate the over estimation 


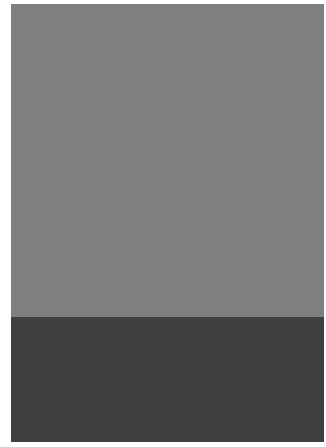

(a)

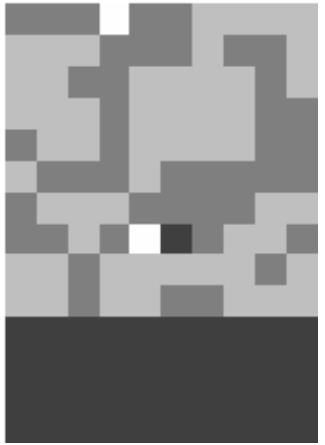

(b)

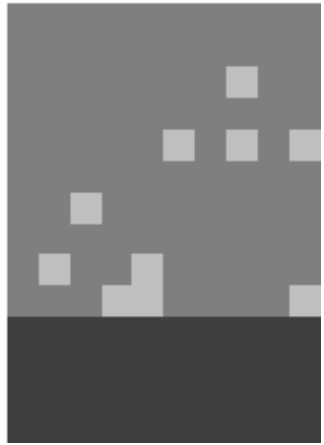

(c)

Fig. 5. Number of tensor in each voxel for experiment in Figure 4. We show the number of tensors coded as grey scale ( dark $=1$, dark grey $=2$, light grey $=3$ and white $=4$ ). (a) Ground truth of synthetic data, (b) estimation by equation (4) and (c) estimation using our proposal in equation (5). The overestimation problem is improved in our formulation.

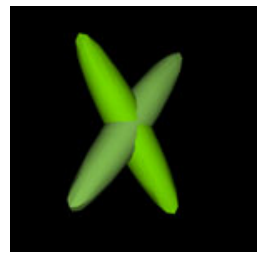

(a)

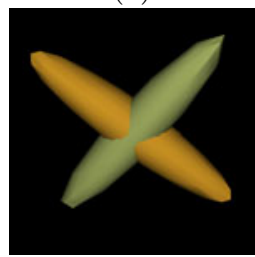

(d)

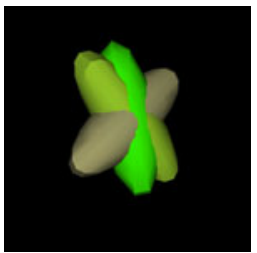

(b)

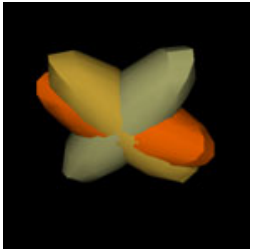

(e)

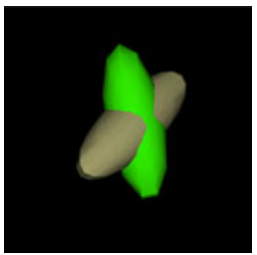

(c)

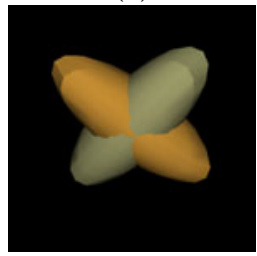

(f)

Fig. 6. Zoom of some voxels of the synthetic data of the Figure 4. (a) and (d) are the GT. (b) and (e) are the results by solving (4). (c) and (f) are the result by using our proposal in (5).

problem of formulation in (4). We select two representative voxels in order to show details of the computed solutions. Figure 6] shows the solutions associated to the two tested formulations.

The Table 1 shows the $D_{F}$ mean error values, the $D_{F}$ error variance and the percentage of voxels that had less error w.r.t the other method for formulations in (4) and (5). We can see that the mean error and the error variance are lower for our proposal. Also, in a $91.43 \%$ of the cases, the new formulation has a lower error. 
Table 1. $D_{F}$ mean and $D_{F}$ variance of the $3 \mathrm{D}$ synthetic data by using (4) and (5)

\begin{tabular}{|l|c|c|c|}
\hline Method & mean error & variance error & lowest error (percentage) \\
\hline \hline Original formulation & 0.7624 & $2.0 \mathrm{e}-03$ & $8.57 \%$ \\
New formulation & 0.2995 & $8.5 \mathrm{e}-04$ & $91.43 \%$ \\
\hline
\end{tabular}

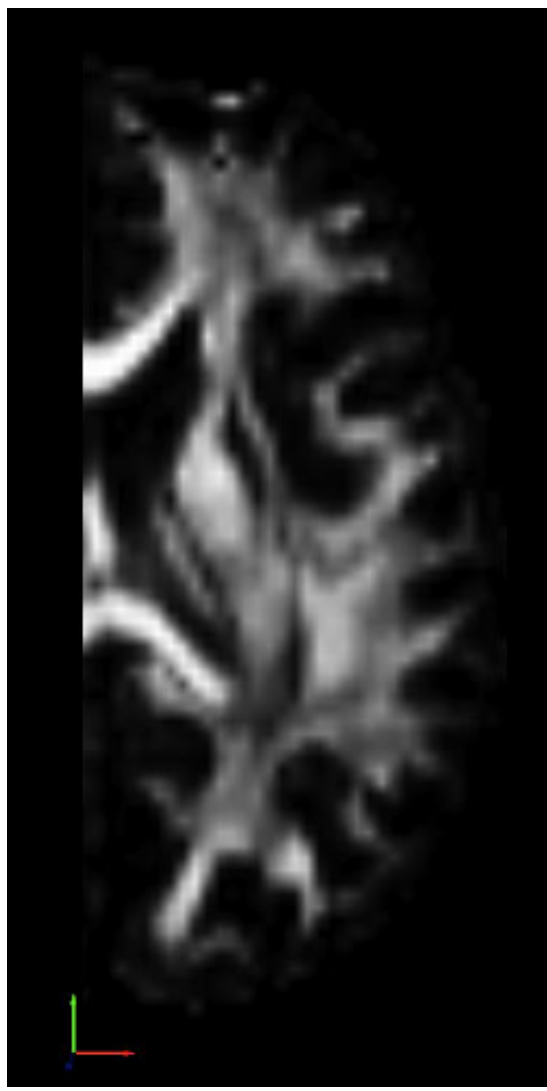

(a)

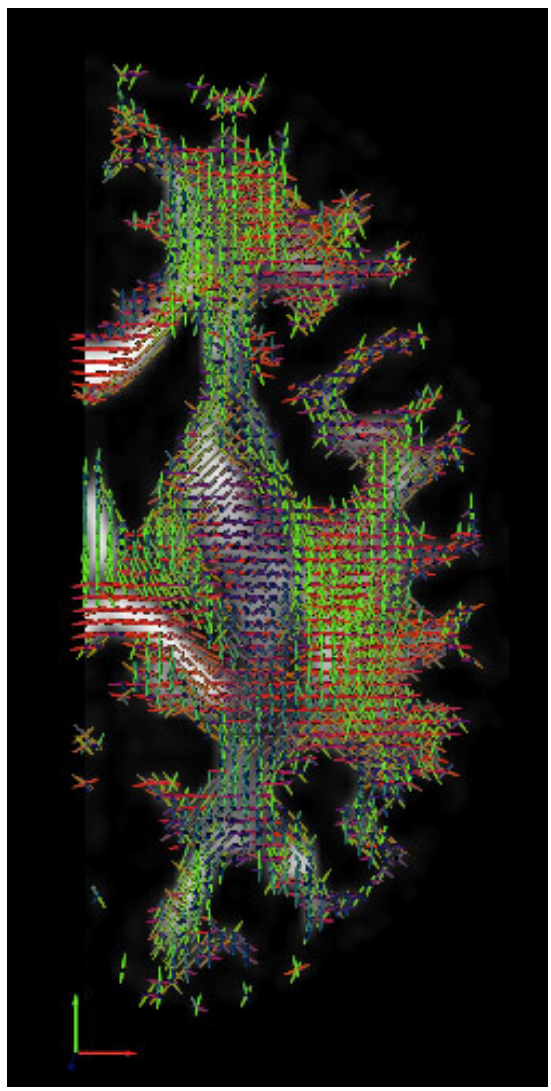

(b)

Fig. 7. Results In vivo Brain Human Data. (a) White matter struct by showing the Fractional Anisotropy map, (b) fiber estimations using the equation 5

\subsection{Real Data}

The last experiment was performed on in vivo Brain Human data, these data have the following features:

- In vivo Brain Human Data: A single healthy volunteer was scanned on a Siemens Trio 3T scanner with 12 channel coil. Acquisition parameters: single-shot echo-planar imaging, five images for $b=0 \mathrm{~s} / \mathrm{mm}, 64 \mathrm{DW}$ images with unique, isotropically distributed orientations $\left(b=1000 \mathrm{~s} / \mathrm{mm}^{2}\right)$, 
$\mathrm{TR}=6700 \mathrm{~ms}, \mathrm{TE}=85 \mathrm{~ms}, 90^{\circ}$ flip angle, voxel dimensions equal to $2 \times 2 \times 2$ $\mathrm{mm}^{3}$. The approximated $\mathrm{SNR}=26$.

The Figure 7 shows the results obtained by using the proposal in brain data. We select a region of interest, such that the center of the image shows crossing axon fibers within the right superior longitudinal fasciculus. The Panel 7(a) shows the white matter struct by showing the Fractional Anisotropy (FA) map [19]. The Panel 7(b) shows the estimated multi-tensors. One can see that our sparse solution still captures the complex multi-fiber brain showing several well-known crossing fibers and bifurcations.

\section{Conclusions}

In this article, we presented a new formulation for Diffusion Basis Functions fitting which improves the original formulation by reducing the overestimation effect on number of estimated fibers due to signal noise. The new formulation adds the Tikhonov's regularization term. The improvements of the proposed method was showed on synthetic and real human brain images.

In addition, we presented a new metric to measure the error between two multi-fiber solutions, we note that it differentiates them properly. This metric models the size compartments of the fibers like piles of earth. Also, the proposed measure takes into account the angular error, the number of the fibers and its distribution in one only formulation based on the Earth Mover's Distance.

\section{References}

1. Buxton, R.B.: Introduction to Functional Magnetic Resonance Imaging: Principles and Techniques, 1st edn. Cambridge University Press (2002)

2. Poldrack, R.A.: A structural basis for developmental dyslexia: Evidence from diffusion tensor imaging. In: Wolf, M. (ed.) Dyslexia, Fluency, and the Brain, pp. 213-233. York Press (2001)

3. Basser, P.J., Mattiello, J., Lebihan, D.: MR Diffusion Tensor Spectroscopy and Imaging. Biophysical Journal 66, 259-267 (1994)

4. Basser, P.J., Pierpaoli, C.: Microstructural and physiological features of tissues elucidated by quantitative-diffusion-tensor MRI. J. Magn. Reson. B 111 (1996)

5. Aranda, R., Rivera, M., Ramírez-Manzanares, A., Ashtari, M., Gee, J.C.: Massive Particles for Brain Tractography. In: Sidorov, G., Hernández Aguirre, A., Reyes García, C.A. (eds.) MICAI 2010, Part I. LNCS, vol. 6437, pp. 446-457. Springer, Heidelberg (2010)

6. Stejskal, E.O.: Use of Spin Echoes in a Pulsed Magnetic-Field Gradient to Study Anisotropic, Restricted Diffusion and Flow. The Journal of Chemical Physics 43, 3597-3603 (1965)

7. Ramírez-Manzanares, A., Rivera, M.: Basis tensor decomposition for restoring intra-voxel structure and stochastic walks for inferring brain connectivity in DT-MRI. Int. Journ. of Comp. Vis. 69, 77-92 (2006)

8. Bergmann, O., Kindlmann, G., Peled, S., Westin, C.F.: Two-tensor fiber tractography. In: IEEE 2007 International Symposium on Biomedical Imaging (ISBI), Washington D.C. (2007) 
9. Malcolm, J.G., Michailovich, O., Bouix, S., Westin, C.F., Shenton, M.E., Rathi, Y.: A filtered approach to neural tractography using the watson directional function. Medical Image Analysis 14, 58-69 (2010)

10. Tuch, D.S., Reese, T.G., Wiegell, M.R., Makris, N., Belliveau, J.W., Wedeen, V.J.: High angular resolution diffusion imaging reveals intravoxel white matter fiber heterogeneity. Magn. Reson. Med. 48, 577-582 (2002)

11. Ramírez-Manzanares, A., Rivera, M., Vemuri, B.C., Carney, P., Mareci, T.: Diffusion basis functions decomposition for estimating white matter intravoxel fiber geometry. IEEE Trans. Med. Imag. 26, 1091-1102 (2007)

12. Ramírez-Manzanares, A., Rivera, M.: Basis pursuit based algorithm for intra-voxel recovering information in DW-MRI. In: Proc. IEEE Sixth Mexican International Conference on Computer Science (ENC 2005), Puebla, México, pp. 152-157 (2005)

13. Jian, B., Vemuri, B.: A unified computational framework for deconvolution to reconstruct multiple fibers from diffusion weighted MRI. IEEE Trans. Med. Imaging (2007)

14. Ramírez-Manzanares, A., Cook, P.A., Gee., J.C.: A comparison of methods for recovering intra-voxel white matter fiber architecture from clinical diffusion imaging scans. Med. Image Comput. Comput. Assist. Interv., 305-312 (2008)

15. Nocedal, J., Wright, S.J.: Numerical optimization. Springer, Heidelberg (1999)

16. Rubner, Y., Tomasi, C., Guibas, L.J.: The Earth Mover's Distance as a Metric for Image Retrieval. International Journal of Computer Vision 40, 99-121 (2000)

17. Pele, O., Werman, M.: Fast and Robust Earth Mover's Distances. In: 2009 IEEE 12th International Conference on Computer Vision, pp. 460-467. IEEE (2009)

18. Pele, O., Werman, M.: A linear Time Histogram Metric for Improved Sift Matching. In: Forsyth, D., Torr, P., Zisserman, A. (eds.) ECCV 2008, Part III. LNCS, vol. 5304, pp. 495-508. Springer, Heidelberg (2008)

19. Le Bihan, D., Mangin, J.F., Poupon, C., Clark, C.A., Pappata, S., Molko, N., Chabriat, H.: Diffusion tensor imaging: Concepts and applications. J. Magn. Reson. Imaging 13, 534-546 (2001) 